\title{
Human taeniasis/cysticercosis: a potentially emerging parasitic disease in Europe
}

\author{
Isaia Symeonidoua , Konstantinos Arsenopoulos ${ }^{a}$, Dimitrios Tzilves $^{\text {b }}$, Barbara Sobac, Sarah Gabriëld, \\ Elias Papadopoulos ${ }^{\mathrm{a}}$
}

Aristotle University of Thessaloniki, Greece; Theageneio Hospital, Thessaloniki, Greece; University of Ljubljana, Slovenia; Ghent University, Merelbeke, Belgium

\begin{abstract}
Taenia saginata (T. saginata)/Taenia solium (T. solium) taeniasis/cysticercosis disease complexes remain a significant challenge for food safety and public health. Human taeniasis is an infectious disease caused by the ingestion of the metacestode larval stage, the cysticerci of T. saginata in beef or T. solium in pork. Humans can also become infected via the ingestion of T. solium eggs. In this case, the cysticerci can establish in the central nervous system, causing the infection called neurocysticercosis. T. solium is of higher importance than T. saginata because the former species can cause neurocysticercosis in humans, a major cause of neurological morbidity in the world. The taeniasis/cysticercosis complex is included in the list of neglected zoonotic diseases by the World Health Organization and Food and Agriculture Organization, with T. solium being the number one foodborne parasite; it occurs mostly in developing countries, such as regions of Asia, Africa and Latin America, where the disease remains endemic. Long absent in Western Europe and other developed countries, cysticercosis has been recently re-emerged as a result of immigration, travel and commerce. In this review, cysticercosis is presented with special emphasis on some aspects of this neglected disease: the main clinical manifestations, risk factors and epidemiology. In addition, any recent advances in diagnostic approaches and treatment are discussed. Finally, the complexities involved in the control of the disease and the need to revise current management strategies are highlighted.
\end{abstract}

Keywords Taeniasis/cysticercosis, Taenia solium, Taenia saginata, neurocysticercosis

Ann Gastroenterol 2018; 31 (4): 1-7

\section{Introduction}

The taeniasis/cysticercosis complex, caused by Taenia solium (T. solium), represents a group of zoonotic diseases that

${ }^{a}$ Laboratory of Parasitology and Parasitic Diseases, School of Veterinary Medicine, Faculty of Health Sciences, Aristotle University of Thessaloniki, Greece (Isaia Symeonidou, Konstantinos Arsenopoulos, Elias Papadopoulos); ${ }^{\mathrm{b}}$ Gastroenterology Department, Theageneio Hospital, Thessaloniki, Greece (Dimitrios Tzilves); 'Institute of Microbiology and Immunology, Faculty of Medicine, University of Ljubljana, Slovenia (Barbara Soba); ${ }^{\mathrm{d} D e p a r t m e n t ~ o f ~ V e t e r i n a r y ~}$ Public Health and Food Safety, Faculty of Veterinary Medicine, Ghent University, Merelbeke, Belgium (Sarah Gabriël)

\section{Conflict of Interest: None}

Correspondence to: Elias Papadopoulos, Laboratory of Parasitology and Parasitic Diseases, School of Veterinary Medicine, Faculty of Health Sciences, Aristotle University of Thessaloniki, PO Box: 393, GR 54124, Thessaloniki, Greece, e-mail: eliaspap@vet.auth.gr

Received 29 November 2017; accepted 30 January 2018; published online 20 April 2018

DOI: https://doi.org/10.20524/aog.2018.0260 are of great importance to public health and are still endemic in developing countries of Africa, Latin America and Asia [1,2]. In non-endemic countries, such as many European countries, the USA, Canada and Australia, cysticercosis is potentially re-emerging through the growing popularity of pork consumption, travel and the increased flow of an immigrant workforce [2-4]. Indeed, a total of 751 patients were reported from 1985-2011 in different countries of Western Europe [3], while a particular epidemic situation is present in Spain and Portugal, as demonstrated by a review of the literature [2]. Taenia saginata (T. saginata) has a global distribution, leads to important economic losses, especially in the meat sector, and represents a food safety issue rather than a serious public health problem, as taeniasis rarely leads to serious clinical signs and symptoms [5]. T. solium and T. saginata are of considerable medical and/or veterinary significance, as they are causative agents of high morbidity and mortality in humans and losses in animal production, mainly because of the condemnation and exclusion of infected carcasses from the human food chain $[1,4]$. Another Taenia species also exists, namely T. asiatica, which is of minor significance to Europe, at least at the present time [6]. These three parasites are responsible 
for two distinct diseases in humans, taeniasis (adult forms) and cysticercosis (larval forms), while in animals (pigs and cattle) they cause only cysticercosis [7].

\section{Mode of infection}

Humans can become infected with T. solium (pork tapeworm) after consumption of raw or insufficiently cooked pork meat harboring the larval stage of this tapeworm, which subsequently develops to the adult stage in the human small intestine. Likewise, T. saginata (beef tapeworm) infection occurs after eating raw or undercooked beef meat carrying the larval stage of the parasite, which again matures to the adult stage in the human small intestine [1]. Additionally, T. solium infection in humans can occur through the accidental ingestion of the eggs of this parasite (fecal-oral route), in a similar way to the mode of infection of the intermediate hosts $[1,8]$. In the latter case, infection with T. solium can result in the formation of cysticerci (larval stage) in the human body, which are of major importance when located in the central nervous system (neurocysticercosis, NCC). Human NCC is the most common parasitic disease of the central nervous system and constitutes a major concern in endemic regions of Latin America, India, Asia, Africa and Eastern Europe [9]. NCC accounts for about 50,000 deaths per year $[10,11]$ and is currently considered to be the most common cause of acquired epilepsy in the developing world, with an estimated 2 million people being affected $[1,12]$. Unfortunately, more cases are being reported in Europe nowadays, mostly as a result of migration [13]. T. solium is the most important foodborne parasite globally $[14,15]$.

Individuals infected with the adult tapeworms, T. solium or T. saginata, expel the proglottids containing the eggs (Fig. 1) of these parasites in their feces. When defecation takes place in the fields, the eggs are spread by water, wind, animal feet, and insects, or by several other means, and contaminate the pasture at varying distances. Cattle usually become infected by ingesting the contaminated herbage. Pigs, being coprophagic animals, usually acquire infection by direct consumption of human feces, or indirectly via contaminated environments. When ingested, the eggs liberate hexacanth embryos or oncospheres in the gastrointestinal tract. Oncospheres enter the bloodstream after crossing the intestinal wall and mature into metacestodes, which are found in usual intermediate host tissues, especially striated muscles (Fig. 2) or the central nervous system, depending on the species of Taenia. Metacestodes then evolve into cysticerci, which are vesicles containing an invaginated scolex $[7,10]$. The life span of a T. solium tapeworm is difficult to determine, but is probably only a few years $[16,17]$ and differs critically from that of T. saginata, which is longer $[1,18]$. The long survival of adult pork tapeworms in humans enhances the possibility for dissemination of eggs to a parasite-naive population [19]. Moreover, the adult T. solium tapeworm has a very high biotic potential, meaning that a single worm can infect many hosts in its surroundings. Consequently, the tapeworm carrier is an important target for control interventions [20]. The most important concepts regarding the mode of infection of taeniasis/cysticercosis disease complexes are summarized in Table 1.

\section{Main clinical manifestations}

Infection with adult Taenia spp. tapeworms may be asymptomatic, with spontaneous release of proglottids for T. saginata, considered the only primary pathognomonic sign. The associated symptoms are often non-specific and include weight loss, abdominal pain, vomiting, diarrhea, constipation and altered appetite. Toxic effects due to the tapeworm's metabolites have also been reported $[6,13]$.

The clinical manifestations of NCC can vary from asymptomatic infection to severe disease and death. Signs and symptoms of NCC are usually nonspecific [21]. Disease severity is indicative of the infection characteristics (size, number and location of cysts). One of the major determinants of the clinical manifestations of NCC is whether the parasites are located in the brain parenchyma or in the extraparenchymal spaces [22]. In parenchymal NCC the most frequently reported symptom

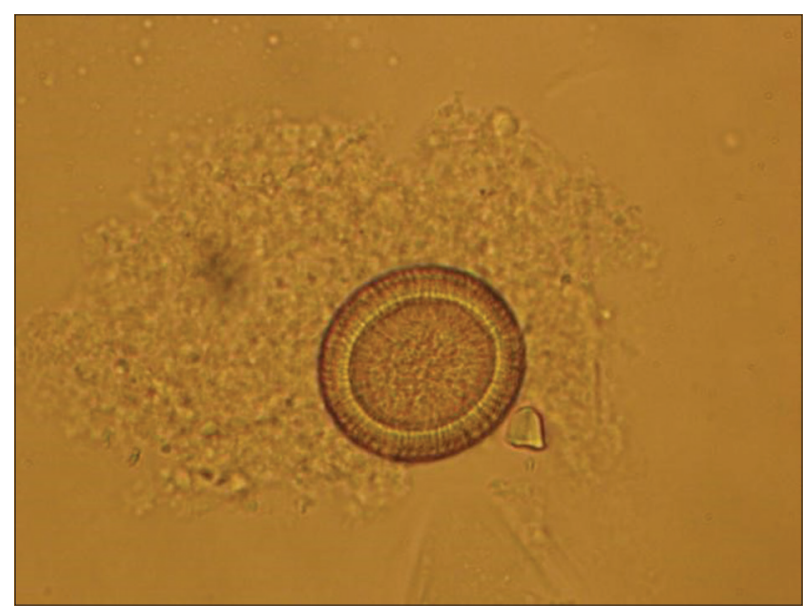

Figure 1 Taenia spp. egg found in human feces (E. Papadopoulos)

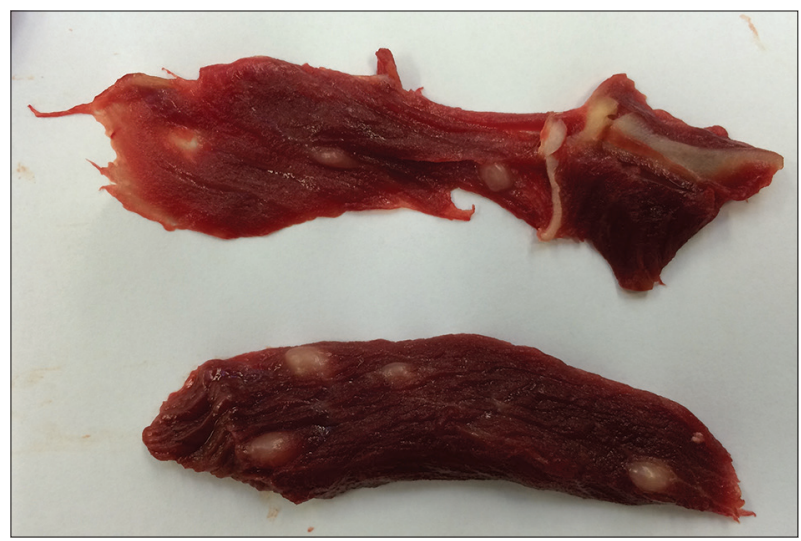

Figure 2 Cysticerci in meat (S. Gabriël) 
Table 1 The most important concepts regarding the mode of infection of taeniasis/cysticercosis disease complexes

Taenia saginata (T. saginata) / Taenia solium (T. solium) taeniasis/cysticercosis disease complexes remain a significant challenge for food safety and public health

Taeniasis in humans occurs due to the ingestion of the metacestode larval stage, the cysticerci of T. saginata in beef or T. solium in pork

Humans also become infected via the ingestion of T. solium eggs and the cysticerci can establish in the central nervous system causing neurocysticercosis (NCC)

NCC is a major cause of neurological morbidity in the world and its prevalence may be on the rise recently as a result of immigration, travel, and commerce

is epilepsy. In a systematic review, Carabin et al reported that epileptic seizures, frequently generalized, were common in up to $80 \%$ of patients with symptomatic NCC [23]. Epileptic seizures occur in two settings, either as acute symptomatic seizures or as "chronic epilepsy" with calcified lesions [24,25]. Another symptom is a recurrent or chronic tension-type headache, attributed to raised intracranial pressure [26]. These headaches are more prevalent than epilepsy and diminish quality of life [23]. In extraparenchymal NCC, cysticerci may reside in the ventricular system or the subarachnoid space. Thus, a lifethreatening acute intracranial hypertension may develop. If cysts lodge in the fourth ventricle, obstruction of cerebrospinal fluid (CSF) flow causes hydrocephalus. Extraparenchymal NCC is subdivided into four groups: ventricular NCC, subarachnoid NCC of the brain convexity, subarachnoid NCC of the lateral fissure and basal subarachnoid NCC, according to the different locations [27].

Whilst in humans the neuropathological and clinical aspects have been well studied and documented, there is limited information on the symptomatology in animals. Recently, a study demonstrated that it is possible for pigs with NCC to develop clinical signs and suffer from seizures like humans with symptomatic NCC [28].

\section{Diagnostic approaches}

Because of the very different disease potentials of the two Taenia species, i.e., T. solium and T. saginata, their accurate identification becomes of vital significance for control and a number of diagnostic methods have been used for this. Each method has advantages and disadvantages. Microscopic, immunological and molecular methods have been used to detect and differentiate between Taenia spp., while a combination of two or more methods appears to provide higher sensitivity $[29,30]$.

For many years, the diagnosis of taeniasis has been based on the detection of eggs by microscopic examination, which still remains the routine in most laboratories [31]. However, given the great morphological similarity among taeniid eggs, identification of species lacks specificity [7,31,32]. Moreover, the morphological examination of gravid proglottids based on internal structures (i.e., the number of uterine branches) relies heavily on them being intact; therefore, it does not always allow accurate species identification. Thus, alternative methods are required for the differentiation of human Taenia spp. causing taeniasis in humans [32].
Regarding immunological assays used to assess the epidemiology of taeniasis, the enzyme-linked immunosorbent assay (ELISA) for coproantigen detection, i.e., adult parasite antigens in human stool, was developed and evaluated by several researchers $[33,34]$. Although this test offers a higher sensitivity and specificity than microscopic diagnosis, it does not support differentiation of T. saginata from T. solium [30,35]. Recently, a protocol was developed that demonstrated good sensitivity and improved specificity for the detection of T. solium antigen in feces [36]. However, in general, these tests were developed in individual laboratories using in-house reagents and have not been independently validated, commercialized and widely used in other diagnostic laboratories [31,37]. Another immunodiagnostic approach for the diagnosis of taeniasis is the detection of antibodies in blood serum [31]. Serological assays mainly use recombinant antigens, such as rTSES33 and rTSES38 [38,39], but are not yet commercially available [31].

Nowadays, the development of molecular tools allows the differential diagnosis between T. saginata and T. solium $[30,40,41]$. A number of molecular methods using polymerase chain reaction (PCR)-based technologies have been developed to either determine the presence of Taenia species-specific DNA in human stools or discriminate between different Taenia spp. [42]. These PCR-based assays display excellent sensitivity and specificity, which are related to the molecular targets [31]. In particular, markers as repetitive sequences of ribosomal DNA, mitochondrial DNA and Antigen 2 gene have proven valuable for revising the taxonomy of the genus [30,43-47].

Regarding NCC, accurate diagnosis is very challenging, especially in developing countries, in the absence of neuroimaging methods or appropriate immunological tests [48]. Moreover, variability in the neuropathology and clinical presentation of the disease makes it often difficult to diagnose [49]. The diagnosis of NCC relies on neuroimaging studies, antibody detection in the serum and CSF, and laboratory analysis of the CSF [50]. Today, a wide variety of immunodiagnostic tests, both commercial and "in-house" have been developed, with various levels of performance [31,51]. The most reliable assay for the detection of antibodies specific for T.solium in serum is the enzyme-linked immunoelectrotransfer blot (EITB) assay, which uses an enriched fraction of glycoproteins from T. solium of porcine origin [52]. EITB assay has a reported specificity and sensitivity of $100 \%$ and $98 \%$, respectively, for patients with two or more viable cysts in the nervous system [53]. However, since serum antibody detection may yield a positive result either in patients with extraneural cysticercosis or in those exposed but without development of 
the infection, results from all tests based on antibody detection should be evaluated with caution [52]. Among antigen-capture assays, especially those conducted on CSF, the HP10 and the B158/B60 monoclonal antibody systems $[54,55]$ are specific and are used in epidemiological studies in endemic regions [56], while they facilitate identification and follow up of complicated NCC [57]. Molecular techniques, such as conventional and real-time PCR, using as matrices either CSF or tissue from biopsy, are specific and relatively sensitive [31,58,59]. In particular, PCR amplification of the pTsol9 repetitive element of the parasite genome in the CSF of patients exhibited the highest sensitivity (95.9\%) and variable specificity (80\% up to $100 \%$ ), depending on the controls used [60]. Nowadays, a set of diagnostic criteria proposed by Del Brutto is widely accepted and has been proven valuable for the diagnosis and management of NCC in patients living in endemic, as well as in non-endemic countries [49]. For animal cysticercosis, the diagnosis is based mainly on classical diagnostic tools such as tongue or meat inspection, which remains the gold standard, and serology for pigs [61].

\section{Treatment}

NCC management can be challenging; it requires a multidisciplinary team, including neurologists, radiologists, parasitologists, etc., and should be individualized, as current options are limited [10]. Treatment of symptoms remains the cornerstone of NCC management and involves anti-inflammatories, antiepileptic drugs, analgesics, and management of intracranial hypertension if present [62]. Antiparasitic therapy is to be considered later on, when the patient is stable without symptoms, and mainly for active disease $[10,63]$. Its aim is the destruction of cysts and the reduction of the number of active lesions and long-term seizure frequency. However, administration of antiparasitic drugs can lead to inflammation due to cyst degeneration and thus increase cerebral edema. Therefore, such treatment should be combined with anti-inflammatory medication [64]. The efficacy of antiparasitic treatment is partial and strategies to improve treatment regimens are warranted, as well as new drugs with better pharmacokinetic profiles [10]. One course of such treatment has been reported to yield a $70 \%$ resolution of cysts vs. $40 \%$ in untreated patients $[65,66]$. Albendazole and praziquantel are the two most common antiparasitic agents used, with albendazole ( $15 \mathrm{mg} / \mathrm{kg}$ per day for 8 days) being the antiparasitic agent of choice in NCC, since it displays a greater cysticidal effect, a lower price and less interaction with other drugs $[66,67]$. Moreover, albendazole is well tolerated and seems to decrease both long-term seizure frequency and the number of cysts $[24,27,67]$. Furthermore, combined treatment with both albendazole and praziquantel has resulted in an enhanced cysticidal effect in patients with multiple cerebral cysts, while the side effects did not increase [68].

The treatment of taeniasis includes a single administration of praziquantel $(5-10 \mathrm{mg} / \mathrm{kg}$ ) or niclosamide (adults and children over 6 years: $2 \mathrm{~g}$, single-administration after a light breakfast, followed after $2 \mathrm{~h}$ by a laxative; children aged 2-6 years: $1 \mathrm{~g}$; children under 2 years: $500 \mathrm{mg}$ ) [69]. Praziquantel, though more effective, brings a risk of inducing epileptic seizures or convulsions in carriers with concurrent asymptomatic NCC, whereas niclosamide, not absorbed by the intestine, is safer, although not available in many endemic countries $[5,70]$.

\section{Control of cysticercosis}

Although many attempts have been made, eradication of this zoonosis is difficult and has been fully achieved only in few countries [9]. This is for several reasons, such as the existence of free-roaming pig production, a lack of basic sanitation, and home slaughter without meat inspection $[1,71]$. Furthermore, the high fecundity and extended longevity of adult parasites exacerbate the challenge of breaking the cycle for transmission. Additionally, the eggs are highly resistant to environmental conditions, remaining viable for up to 8 months or more in warm and humid climates [4]. Another strong limitation is the lack of an effective multi-sectorial approach, including public health, animal health and environment [72]. Finally, the epidemiological data obtained on T. solium cysticercosis do not reflect the accurate picture of disease prevalence, while solid field data evaluating the different intervention tools are lacking $[72,73]$. All the above tend to mask the recognition of the depth of the economic and medical impacts associated with human taeniasis/cysticercosis [73].

Effective control of taeniasis/cysticercosis is challenging and relies on targeting all disease risk factors, along with public education on the health issues, especially in endemic regions $[49,71]$. Towards this direction a computer-based T. solium education tool has been developed [71]. Cutting off the life cycle of $T$. solium depends on improvements in public sanitation and pig husbandry [5,74]. Pig-rearing conditions are believed to be the main risk factor for T. solium transmission [72]. Even though the free-roaming system offers an economic advantage to breeders of smallholder pig farms in non-industrialized rearing conditions, pigs should be kept indoors without any contact with human feces [75]. Another approach for tight control of the disease is meat inspection in order to condemn infected carcasses or treat them accordingly (i.e., by freezing at $-10^{\circ} \mathrm{C}$ for 10 days, or heating above $60^{\circ} \mathrm{C}$ ) [76]. In the European Union, the requirements for ante- and postmortem inspection of animals for human consumption, including cattle and pigs, are laid out in regulation 854/2004 [76]. In the same frame, people must be educated to consume only well cooked pork meat [77].

Broader control strategies for developing countries may include massive and sustained chemotherapy for humans, using praziquantel, niclosamide or albendazole $[5,78,79]$. Notably, commercialized chemotherapy of pigs with a single dose of oxfendazole has been shown to treat porcine T. solium infections effectively, in terms of interrupting the life cycle of the parasite for at least 3 months in field conditions [80,81].

Another attractive option is the vaccination of pigs and cattle against $T$. solium and $T$. saginata, respectively. In contrast 
Table 2 The most important concepts regarding the diagnosis, treatment, and control of taeniasis/cysticercosis disease complexes Microscopic, immunological and molecular methods have been used to detect and differentiate between Taenia spp., while a combination of two or more methods provides higher sensitivity

The diagnosis of neurocysticercosis (NCC) is challenging and relies upon neuroimaging studies, antibody detection in the serum and cerebrospinal fluid, and laboratory analysis of the cerebrospinal fluid

Symptomatic treatment remains the cornerstone of NCC management and involves anti-inflammatories, antiepileptic drugs, analgesics, and management of intracranial hypertension, if present

Effective control of taeniasis/cysticercosis depends on targeting all disease risk factors, along with increasing public awareness, especially in endemic regions

Broader control strategies for developing countries include massive and sustained chemotherapy of humans and vaccination of pigs and cattle against Taenia solium and Taenia saginata, respectively

to cattle immunization, which is less important and not yet commercialized, pig immunization, using recombinant antigens, has attracted interest and demonstrated diverse degrees of protection [5,82]. Two major vaccine candidates against porcine cysticercosis are now available: S3Pvac and TSOL18 [83,84]. The TSOL18 vaccine has proven to be very effective under controlled field trials, since two doses induce almost complete protection against the development of parasites in all vaccinated pigs [85]. TSOL18 vaccine is now being commercialized and further tested under natural conditions of transmission [5]. However, the need for at least 2 doses and cold chain, and the setting up of a distribution network might hamper the implementation.

Overall, it should be stressed that to reach a substantial and sustained reduction of transmission and morbidity, multiple tools will have to be combined for a long period [5]. The most important concepts regarding the diagnosis, treatment and control of taeniasis/cysticercosis disease complexes are summarized in Table 2.

\section{Concluding remarks and future perspectives}

In spite of considerable scientific progress, our knowledge of the taeniasis/cysticercosis complex is still modest and the challenges ahead in endemic areas are enormous. Recently, several European groups joined forces in a European network on taeniasis/cysticercosis, CYSTINET (COST Action TD1302), to address these challenges and develop guidelines for the management of taeniasis and NCC, including diagnostics, patient management and follow-up. Further research is needed to lead to the development of more effective tools for diagnosis. Advanced immunological and molecular markers are the baseline for modern diagnostics and should be combined with the analysis of spatial patterns of distribution for evidencebased control. Additionally, future studies should attempt to determine whether immune system differences due to genetic diversity among human populations (European, African, American and Asian) affect the clinical outcome of the disease. Towards control of these zoonoses, we have to clarify the exact risk factors and their distribution in space and time. This can be achieved by integrating human and animal epidemiology with parasite ecology and host behavior. Strategies such as the One Health concept and cooperation through public and private partnerships play a crucial role in ensuring that the global control is improving. Another important aspect is the relevant education of health professionals, so that cases of taeniasis and NCC are diagnosed and managed in a correct way, thus decreasing their impact on public health. A major hope resides in the genomes and the transcriptomes of tapeworms, which have recently been revealed. Next-generation sequencing and computational analysis can provide research-based evidence towards evolutionary biology, host-parasite relationship and immunomodulation. Furthermore, this solid molecular basis will provide insights into identification of key excretorysecretory proteins to advance the fields of diagnosis, vaccination, and therapy.

\section{Acknowledgment}

The work was carried out within the frame of the Cost Action TD1302 "European Network on Taeniasis/Cysticercosis (CYSTINET)”.

\section{References}

1. Ito A, Yanagida T, Nakao M. Recent advances and perspectives in molecular epidemiology of Taenia solium cysticercosis. Infect Genet Evol 2016;40:357-367.

2. Fabiani S, Bruschi F. Neurocysticercosis in Europe: still a public health concern not only for imported cases. Acta Trop 2013;128:18-26.

3. Del B OH. Neurocysticercosis in Western Europe: a re-emerging disease? Acta Neurol Belg 2012;112:335-343.

4. Bobes RJ, Fragoso G, Fleury A, et al. Evolution, molecular epidemiology and perspectives on the research of taeniid parasites with special emphasis on Taenia solium. Infect Genet Evol 2014;23:150-160.

5. Okello AL, Thomas LF. Human taeniasis: current insights into prevention and management strategies in endemic countries. Risk Manag Healthc Policy 2017;10:107-116.

6. Ito A, Nakao M, Wandra T. Human Taeniasis and cysticercosis in Asia. Lancet 2003;362:1918-1920.

7. Hoberg EP. Taenia tapeworms: their biology, evolution and socioeconomic significance. Microbes Infect 2002;4:859-866.

8. Sotelo J, Del Brutto OH. Brain cysticercosis. Arch Med Res 2000;31:3-14. 
9. Garcia HH, Del B OH. Taenia solium cysticercosis. Infect Dis Clin North Am 2000;14:97-119.

10. Fogang YF, Savadogo AA, Camara M, et al. Managing neurocysticercosis: challenges and solutions. Int $J$ Gen Med 2015;8:333-344.

11. Hoberg EP. Phylogeny of Taenia: Species definitions and origins of human parasites. Parasitol Int 2006;55(Suppl):S23-S30.

12. Raoul F, Li T, Sako Y, et al. Advances in diagnosis and spatial analysis of cysticercosis and taeniasis. Parasitology 2013;140:1578-1588.

13. Dorny P, Praet N. Taenia saginata in Europe. Vet Parasitol 2007;149:22-24.

14. Robertson LJ, van der Giessen JW, Batz MB, Kojima M, Cahill S. Have foodborne parasites finally become a global concern? Trends Parasitol 2013;29:101-103.

15. Robertson LJ, Sprong H, Ortega YR, van der Giessen JW, Fayer R. Impacts of globalisation on foodborne parasites. Trends Parasitol 2014;30:37-52.

16. Yoshino K. On the subjective symptoms caused by the parasitism of Taenia solium and its development in man. J Med Assoc Formosa 1934;33:183-194.

17. Lightowlers MW. Control of Taenia solium taeniasis/ cysticercosis: past practices and new possibilities. Parasitology 2013;140:1566-1577.

18. Pawlowski ZS. Epidemiology and prevention of Taenia saginata infection. In: Flisser A, Willms K, Laclette JP, Larralde C, Ridaura C, Beltran F (editors): Cysticercosis. Present state of knowledge and perspective. Academic Press: New York; 1982, pp. 69-85.

19. Smith JL. Taenia solium neurocysticercosis. J Food Prot 1994;57:831-844.

20. Del Brutto OH, Garcia HH. Control and perspectives for elimination of Taenia solium taeniosis/cysticercosis. In: Del Brutto OH, Garcia HH (editors): Cysticercosis of the human nervous system. Springer: Berlin; 2014, pp. 125-135.

21. Garcia HH, Del Brutto OH; Cysticercosis Working Group in Peru. Neurocysticercosis: updated concepts about an old disease. Lancet Neurol 2005;4:653-661.

22. Estañol B, Corona T, Abad P. A prognostic classification of cerebral cysticercosis: therapeutic implications. J Neurol Neurosurg Psychiatry 1986;49:1131-1134.

23. Carabin H, Ndimubanzi PC, Budke CM, et al. Clinical manifestations associated with neurocysticercosis: a systematic review. PLoS Negl Trop Dis 2011;5:e1152.

24. Nash TE, Garcia HH, Rajshekhar V, Del Brutto OH. Clinical cysticercosis: diagnosis and treatment. In: Murrell KD (editor): WHO/FAO/OIE guidelines for the surveillance, prevention and control of Taeniosis/Cysticercosis. OIE: Paris; 2005, pp. 73-99.

25. Nash TE, Mahanty S, Loeb JA, et al. Neurocysticercosis: a natural human model of epileptogenesis. Epilepsia 2015;56:177-183.

26. Fogang YF, Camara M, Diop AG, Ndiaye MM. Cerebral neurocysticercosis mimicking or comorbid with episodic migraine? BMC Neurol 2014;14:138.

27. Nash TE, Garcia HH. Diagnosis and treatment of neurocysticercosis. Nat Rev Neurol 2011;7:584-594.

28. Trevisan C, Mkupasi EM, Ngowi HA, Forkman B, Johansen MV. Severe seizures in pigs naturally infected with Taenia solium in Tanzania. Vet Parasitol 2016;220:67-71.

29. Mackey TM, Liang BA, Cuomo R, Hafen R, Brouwer KC, Lee DE. Emerging and reemerging neglected tropical diseases: a review of key characteristics, risk factors and the policy and innovation environment. Clinic Microbiol Rev 2014;27:949-979.

30. Praet N, Verweij JJ, Mwape KE, et al. Bayesian modelling to estimate the test characteristics of coprology, coproantigen ELISA and a novel real-time PCR for the diagnosis of taeniasis. Trop Med Int Health 2013;18:608-614.

31. Gómez-Morales MA, Gárate T, Blocher J, et al. Present status of laboratory diagnosis of human taeniosis/cysticercosis in Europe. Eur J Clin Microbiol Infect Dis 2017;36:2029-2040.

32. Dorny P, Brandt J, Geerts S. Detection and diagnosis. In: Murrell KD (editor): WHO/FAO/OIE guidelines for the surveillance, prevention and control of taeniosis/cysticercosis. OIE: Paris; 2005, pp. 45-55.

33. Sako Y, Itoh S, Okamto M, Nakaya K, Ito A. Simple and reliable preparation of immunodiagnostic antigens for Taenia solium cysticercosis. Parasitology 2013;140:1589-1594.

34. Allan JC, Avila G, Garcia N J, Flisser A, Craig PS. Immunodiagnosis of taeniasis by coproantigen detection. Parasitology 1990;101:473-477.

35. Tembo A, Craig PS. Taenia saginata taeniosis: copro-antigen timecourse in a voluntary self-infection. J Helminthol 2015;89:612-619.

36. Guezala MC, Rodriguez S, Zamora H, et al. Development of a species-specific coproantigen ELISA for human Taenia solium taeniasis. Am J Trop Med Hyg 2009;81:433-437.

37. Jabbar A, Gauci C, Lightowlers MW. Diagnosis of human taeniasis. Microbiol Austral 2016;37:43-45.

38. Levine MZ, Calderón JC, Wilkins PP, et al. Characterization, cloning, and expression of two diagnostic antigens for Taenia solium tapeworm infection. J Parasitol 2004;90:631-638.

39. Handali S, Klarman M, Gaspard AN, et al. Development and evaluation of a magnetic immunochromatographic test to detect Taenia solium, which causes taeniasis and neurocysticercosis in humans. Clin Vaccine Immunol 2010;17:631-637.

40. Mayta H, Talley A, Gilman RH, et al. Differentiating Taenia solium and Taenia saginata infections by simple hematoxylin-eosin staining and PCR-restriction enzyme analysis. J Clin Microbiol 2000;38:133-137.

41. González LM, Montero E, Puente S, et al. PCR tools for the differential diagnosis of Taenia saginata and Taenia solium taeniasis/cysticercosis from different geographical locations. Diagn Microbiol Infect Dis 2002;42:243-249.

42. Ng-Nguyen D, Stevenson MA, Dorny P, et al. Comparison of a new multiplex real-time PCR with the Kato Katz thick smear and coproantigen ELISA for the detection and differentiation of Taenia spp. in human stools. PLoS Negl Trop Dis 2017;11:e0005743.

43. González LM, Montero E, Harrison LJ, Parkhouse RM, Garate T. Differential diagnosis of Taenia saginata and Taenia solium infection by PCR. J Clin Microbiol 2000;38:737-744.

44. Yamasaki H, Allan JC, Sato MO, et al. DNA differential diagnosis of taeniasis and cysticercosis by multiplex PCR. J Clin Microbiol 2004;42:548-553.

45. Sato MO, Sako Y, Nakao M, et al. A possible nuclear DNA marker to differentiate the two geographic genotypes of Taenia solium tapeworms. Parasitol Int 2011;60:108-110.

46. González LM, Bailo B, Ferrer E, et al. Characterization of the Taenia spp HDP2 sequence and development of a novel PCR-based assay for discrimination of Taenia saginata from Taenia asiatica. Parasit Vectors 2010;3:51.

47. Roelfsema JH, Nozari N, Pinelli E, Kortbeek LM. Novel PCRs for differential diagnosis of cestodes. Exp Parasitol 2016;161:20-26.

48. Garcia HH, Nash TE, Del Brutto OH. Clinical symptoms, diagnosis, and treatment of neurocysticercosis. Lancet Neurol 2014;13:1202-1215.

49. Del Brutto OH. Diagnostic criteria for neurocysticercosis, revisited. Pathog Glob Health 2012;106:299-304.

50. Takayanagui OM, Odashima NS. Clinical aspects of neurocysticercosis. Parasitol Int 2006;55 Suppl: S111-S115.

51. Carod JF, Randrianarison M, Razafimahefa J, et al. Evaluation of the performance of 5 commercialized enzyme immunoassays for the detection of Taenia solium antibodies and for the diagnosis of neurocysticercosis. Diagn Microbiol Infect Dis 2012;72:85-89.

52. Deckers N, Dorny P. Immunodiagnosis of Taenia solium taeniosis/ 
cysticercosis. Trends Parasitol 2010;26:137-144.

53. Tsang VC, Brand JA, Boyer AE. An enzyme-linked immunoelectrotransfer blot assay and glycoprotein antigens for diagnosing human cysticercosis (Taenia solium). J Infect Dis 1989;159:50-59.

54. Harrison LJ, Joshua GW, Wright SH, Parkhouse RM. Specific detection of circulating surface/secreted glycoproteins of viable cysticerci in Taenia saginata cysticercosis. Parasite Immunol 1989;11:351-370.

55. Van Kerckhoven I, Vansteenkiste W, Claes M, Geerts S, Brandt J. Improved detection of circulating antigen in cattle infected with Taenia saginata metacestodes. Vet Parasitol 1989;76:269-274.

56. Gabriël S, Blocher J, Dorny P, et al. Added value of antigen ELISA in the diagnosis of neurocysticercosis in resource poor settings. PLoS Negl Trop Dis 2012;6:e1851.

57. Fleury A, Garcia E, Hernández M, et al. Neurocysticercosis: HP10 antigen detection is useful for the follow-up of the severe patients. PLoS Negl Trop Dis 2013;7:e2096.

58. Yera H, Dupont D, Houze S, et al. Confirmation and follow-up of neurocysticercosis by real-time PCR in cerebrospinal fluid samples of patients living in France. J Clin Microbiol 2011;49:4338-4340.

59. Hernández M, Gonzalez LM, Fleury A, et al. Neurocysticercosis: detection of Taenia solium DNA in human cerebrospinal fluid using a semi-nested PCR based on HDP2. Ann Trop Med Parasitol 2008; 102:317-323.

60. Michelet L, Fleury A, Sciutto E, et al. Human neurocysticercosis: comparison of different diagnostic tests using cerebrospinal fluid. J Clin Microbiol 2011;49:195-200.

61. Dorny P, Phiri IK, Vercruysse J, et al. A Bayesian approach for estimating values for prevalence and diagnostic test characteristics of porcine cysticercosis. Int J Parasitol 2004;34:569-576.

62. Nash TE, Singh G, White AC, et al. Treatment of neurocysticercosis: current status and future research needs. Neurology 2006;67:1120-1127.

63. Winkler AS, Richter H. Landscape analysis: management of neurocysticercosis with an emphasis on low and middle-income countries. World Health Organization: Geneva; 2015.

64. Takayanagui OM, Odashima NS, Bonato PS, Lima JE, Lanchote VL. Medical management of neurocysticercosis. Expert Opin Pharmacother 2011;12:2845-2856.

65. Garcia HH, Pretell EJ, Gilman RH, et al; Cysticercosis Working Group in Peru. A trial of antiparasitic treatment to reduce the rate of seizures due to cerebral cysticercosis. $N$ Engl J Med 2004;350:249-258.

66. Del Brutto OH, Roos KL, Coffey CS, García HH. Meta-analysis: Cysticidal drugs for neurocysticercosis: albendazole and praziquantel. Ann Intern Med 2006;145:43-51.

67. Matthaiou DK, Panos G, Adamidi ES, Falagas ME. Albendazole versus praziquantel in the treatment of neurocysticercosis: a metaanalysis of comparative trials. PLoS Negl Trop Dis 2008;2:e194.

68. Garcia HH, Gonzales I, Lescano AG, et al; Cysticercosis Working Group in Peru. Efficacy of combined antiparasitic therapy with praziquantel and albendazole for neurocysticercosis: a double-blind, randomised controlled trial. Lancet Infect Dis
2014;14:687-695.

69. Pawlowski Z, Allan JC, Meinardi H. Control measures for taeniosis and cysticercosis. In: Murrell KD (editor): WHO/FAO/OIE guidelines for the surveillance, prevention and control of taeniosis/ cysticercosis. OIE: Paris; 2005, pp. 73-99.

70. Li T, Ito A, Chen X, et al. Usefulness of pumpkin seeds combined with areca nut extract in community-based treatment of human taeniasis in northwest Sichuan Province, China. Acta Trop 2012;124:152-157.

71. Johansen MV, Trevisan C, Braae UC, et al. The Vicious Worm: a computer-based Taenia solium education tool. Trends Parasitol 2014;30:372-374.

72. Devleesschauwer B, Allepuz A, Dermauw V, et al. Taenia solium in Europe: Still endemic? Acta Trop 2017;165:96-99.

73. Laranjo-González M, Devleesschauwer B, Trevisan C, et al. Epidemiology of taeniosis/cysticercosis in Europe, a systematic review: Western Europe. Parasit Vectors 2017;10:349.

74. Gabriël S, Dorny P, Mwape KE, et al. Control of Taenia solium taeniasis/cysticercosis: The best way forward for sub-Saharan Africa? Acta Trop 2017;165:252-260.

75. Taylor MA, Coop RL, Wall RL. Veterinary Parasitology. Blackwell Publishing: Oxford; 2007.

76. European Commission. Commission Regulation (EU) No 854/2004: laying down specific rules for the organization of official controls on products of animal origin intended for human consumption. Union TEPatCotE: Brussels; 2004.

77. Alexander A, John KR, Jayaraman T, et al. Economic implications of three strategies for the control of taeniasis. Trop Med Int Health 2011;16:1410-1416.

78. Thomas LF. Landscape Analysis: Control of Taenia solium. World Health Organization: Geneva; 2015.

79. Garcia HH, Gonzalez AE, Gilman RH, et al; Cysticercosis Working Group in Peru. Combined human and porcine mass chemotherapy for the control of T. solium. Am J Trop Med Hyg 2006;74:850-855.

80. Gonzalez AE, Gavidia C, Falcon N, et al; Cysticercosis Working Group in Peru. Protection of pigs with cysticercosis from further infections after treatment with oxfendazole. Am J Trop Med Hyg 2001;65:15-18.

81. Sikasunge CS, Johansen MV, Willingham AL $3^{\text {rd }}$, Leifsson PS, Phiri IK. Taenia solium porcine cysticercosis: viability of cysticerci and persistency of antibodies and cysticercal antigens after treatment with oxfendazole. Vet Parasitol 2008;158:57-66.

82. Gonzalez AE, Gauci CG, Barber D, et al. Vaccination of pigs to control human neurocysticercosis. Am J Trop Med Hyg 2005;72:837-839.

83. Lightowlers MW. Eradication of Taenia solium cysticercosis: a role for vaccination of pigs. Int J Parasitol 2010;40:1183-1192.

84. Sciutto E, Fragoso G, Hernández M, et al. Development of the S3Pvac vaccine against porcine Taenia solium cysticercosis: a historical review. J Parasitol 2013;99:686-692.

85. Assana E, Kyngdon CT, Gauci CG, et al. Elimination of Taenia solium transmission to pigs in a field trial of the TSOL18 vaccine in Cameroon. Int J Parasitol 2002;40:515-519. 